\title{
Infarto do miocárdio induzido por cocaína
}

Cocaine-induced myocardial infarction

\begin{tabular}{l|l}
\multicolumn{1}{c|}{ unitermos } & res U MO \\
\hline Cocaína & $\begin{array}{l}\text { Com os aumentos da difusão e da facilidade de acesso à cocaína, registra-se aumento da morbidade e } \\
\text { da mortalidade associadas ao seu uso. O seu consumo tem sido associado a doenças cardiovasculares } \\
\text { Infarto do miocárdio }\end{array}$ \\
$\begin{array}{l}\text { Necrópsia } \\
\text { consumo, entre elas isquemia e infarto do miocárdio. Desde o primeiro relato de associação entre o } 0 \\
\text { amplamente estudados, a fim de se estabelecerem seus mecanismos fisiopatológicos, alterações } \\
\text { estruturais, diagnóstico, repercussões hemodinâmicas e prognóstico. O objetivo deste estudo foi relatar } \\
\text { um caso de múltiplos infartos do miocárdio induzido pelo uso de cocaína com estudo necroscópico. }\end{array}$
\end{tabular}

The increased distribution and access to cocaine, lead to an increase of morbidity and mortality associated with its use. Cocaine use has been associated with cardiovascular diseases, among them ischemia and myocardial infarction. Since the first report of association between the use of cocaine, myocardial ischemia and infarction in 1982, the cardiovascular effects of cocaine have been extensively studied in order to establish its pathophysiological mechanism, structural changes, methods of diagnosis, prognosis and homodynamic effects. This study reports a case of multiple myocardial infarction induced by the use of cocaine with necroscopic study.

\section{key words}

Cocaine

Myocardial infarction

Autopsy 


\section{Introdução}

O consumo de cocaína tem sido associado a doenças cardiovasculares (DCVs), tanto agudas como crônicas ${ }^{(1)}$. Entre estas estão infarto agudo do miocárdio, isquemia miocárdica (silenciosa ou associada com angina), aceleração do desenvolvimento de aterosclerose, miocardite, cardiomiopatia, arritmias, hipertensão arterial (HA), dissecção aórtica e endocardite ${ }^{(1)}$. Esses eventos podem ocorrer por meio de qualquer via de administração e com grande ou até mesmo pequena quantidade do fármaco(2).

Com o aumento da difusão e a facilidade de acesso à cocaína, registram-se aumentos da morbidade e da mortalidade associadas ao seu uso. Estima-se que nos Estados Unidos da América, 25 milhões de pessoas fizeram uso da cocaína ao menos uma vez, e 5 milhões a usam regularmente ${ }^{(1,2)}$.

Estabelecer a incidência de infarto do miocárdio associado ao uso de cocaína é, notoriamente, tarefa muito difícil(3). De qualquer forma, qualquer incidência estimada é inaceitável, uma vez que é condição prevenível( ${ }^{(3)}$. Nesse sentido, o conhecimento dos mecanismos fisiopatológicos e o diagnóstico precoce das alterações cardíacas relacionadas com o uso da cocaína são elementos fundamentais para o tratamento adequado e a diminuição da morbidade e mortalidade relacionadas com o consumo da droga.

Embora qualquer usuário esteja em risco, o doente típico é um homem na faixa etária média de 30 anos e com quadro de angina, infarto, arritmias complexas, miocardite, cardiomiopatia, HA, dissecção aórtica e endocardite; tabagismo é um fator de risco comumente associado ${ }^{(1-3)}$. Por conseguinte, a possibilidade do uso abusivo de cocaína deve ser aventada nos pacientes que apresentem tais características clínicas ${ }^{(1,2)}$.

O objetivo deste estudo foi discutir essa entidade nosológica, de crescente importância social, por meio do relato de caso de um infarto do miocárdio induzido pelo uso de cocaína diagnosticado em nosso serviço, descrevendo os achados de história clínica, estratificação diagnóstica e abordagem terapêutica. Por meio da necropsia, buscamos correlacionar todo o quadro clínico e confrontar as alterações estruturais encontradas com as descritas na literatura.

\section{Descrição do caso}

Paciente do sexo masculino, 24 anos, negro, solteiro, com história de tosse, dispneia e emagrecimento de cerca de $15 \mathrm{~kg}$ nas últimas três semanas. Já vinha apresentando, há cerca de seis meses, cansaço aos esforços habituais. Antecedentes de tabagismo e etilismo e usuário de drogas (cocaína). Há 20 dias, com a intensificação do quadro respiratório, procurou serviço municipal de pronto atendimento, onde foi diagnosticado com pneumonia e tratado com amoxicilina. Nessa ocasião, fugiu do hospital e permaneceu em casa, com pouca atividade física, devido à fraqueza. A não melhora o fez procurar nosso serviço de pronto atendimento. Ao exame clínico inicial, foi detectada insuficiência respiratória, e o paciente foi internado para investigação do quadro.

Em algumas horas, houve acentuada piora do quadro respiratório, além de hipotensão e diminuição da perfusão periférica. A frequência cardíaca (FC) estava em 180 bat/min. Foi, então, encaminhado à Unidade de Tratamento Intensivo (UTI) para suporte respiratório. Nessa ocasião, ao exame físico, foi detectada $4^{\circ}$ bulha em ritmo de galope, à ausculta pulmonar sibilios difusos bilateralmente, além de oligúria. A radiografia de tórax revelou infiltrado peri-hilar; o eletrocardiograma, sobrecarga das câmaras direitas; e, ao ecocardiograma, evidenciaram-se miocardiopatia dilatada, refluxo mitral ++/+4, tricúspide e aórtico. Após dois dias de internação, o paciente evoluiu a óbito.

\section{Discussão}

Em 1982, Coleman et al. relataram pela primeira vez associação entre consumo de cocaína, isquemia e infarto do miocárdio ${ }^{(4)}$. Desde seu primeiro relato até os dias atuais, a dor torácica relacionada com o consumo de cocaína vem sendo um crescente problema entre os pacientes jovens que se apresentam aos serviços de pronto atendimento médico ${ }^{(2,4,6,7)}$.

A fisiopatologia da isquemia e do infarto do miocárdico relacionados com o consumo de cocaína é provavelmente multifatorial e decorre de eventos isolados ou da combinação entre aumento da demanda de oxigênio do miocárdio em situações de suprimento limitado ou fixo, vasoconstrição arterial prolongada e agregação plaquetária com subsequente formação de trombo ${ }^{(1-3)}$.

Em seu mecanismo de ação, a cocaína produz estimulação adrenérgica central e periférica, bloqueando a recaptação pré-sináptica de noradrenalina e dopamina, aumentando, assim, suas concentrações pós-sinápticas.

Lange et al. ${ }^{(5)}$ estudaram 45 pacientes submetidos à angiografia coronária para avaliação de dor torácica. $\mathrm{O}$ 
fluxo sanguíneo no seio coronário e o diâmetro das artérias coronárias foram medidos no início do estudo e após administração intranasal de cocaína ou soro fisiológico. Após administração da cocaína, houve diminuição do fluxo sanguíneo em ambas as artérias coronárias e redução do diâmetro das artérias epicárdicas. Ainda, esses efeitos foram bloqueados por fentolamina, um agente bloqueador alfadrenérgico, sugerindo que a estimulação dos receptores alfa é o provável mecanismo dessas alterações(2).

O consumo de cocaína também está associado à ativação de plaquetas, levando a maiores adesividade e agregação plaquetária, que desempenham papel importante no desenvolvimento de trombos coronários ${ }^{(2,3,6)}$.

Paralelamente a esse quadro de redução do suporte de oxigênio, instala-se um outro de aumento da demanda desse gás em decorrência do aumento do trabalho cardíaco. Os efeitos simpatomiméticos de dose única de cocaína demonstraram aumento da pressão arterial sistólica (PAS) em 20 torr, da pressão arterial diastólica (PAD) em 10 torr e da $\mathrm{FC}$ em $30 \mathrm{bat} / \mathrm{min}^{(7)}$. Esses efeitos são equivalentes a atividade física leve e, de forma isolada, não são suficientes para induzir isquemia cardíaca na maioria dos indivíduos( ${ }^{(8)}$. A estimulação dos receptores betaadrenérgicos resulta em aumento do influxo de cálcio nas células do miocárdio, e o estímulo de receptores alfadrenérgicos resulta em aumento de fosfolipase $\mathrm{C}$ e, posteriormente, inositol trifosfato ${ }^{(8)}$. O aumento intracelular em ambos, inositol trifosfato e cálcio, resulta em aumento do inotropismo cardíaco, o qual, por sua vez, contribui para o aumento do trabalho cardíaco e a demanda de oxigênio ${ }^{(7)}$.

Dessa forma, o cenário de aumento da demanda de oxigênio do miocárdio em situações de suprimento limitado ou fixo, vasoconstrição arterial prolongada e agregação plaquetária com subsequente formação de trombo figura o mecanismo fisiopatológico da isquemia e infarto do miocárdio presentes em nosso caso.

O estudo de necropsia revelou o coração aumentado de tamanho, evidenciando dilatação das câmaras com múltiplos infartos antigos. Embora os ventrículos sejam mais intensamente afetados do que os átrios, observou-se que todas as câmaras do coração estavam dilatadas. Ainda, chamamos a atenção para o fato de que a dilatação ventricular esquerda foi tão intensa que a parede do ventrículo esquerdo parece ter a espessura normal ou até mesmo mais fina. Observou-se também trombose em átrio direito. $\mathrm{O}$ miocárdio se apresentou flácido e pálido, e cicatrizes subendocárdicas podem ser visualizadas, caracterizando múltiplos infartos antigos. O endocárdio do ventrículo esquerdo, especialmente no ápice, que normalmente tende a ser espessado, encontra-se fino e com extensa área de necrose. Havia leve insuficiência mitral e triscúspide por dilatação das câmaras cardíacas. As artérias coronárias estavam pérvias, porém com leve aterosclerose. Os pulmões evidenciaram áreas focais de hemorragia difusamente distribuídas.

Microscopicamente, os miócitos cardíacos, especialmente no subendocárdio, demonstraram alterações degenerativas avançadas, caracterizadas por perda de miofibrilas, efeito que dá às células aparência vazia ou vacuolada. Fibroses intersticial

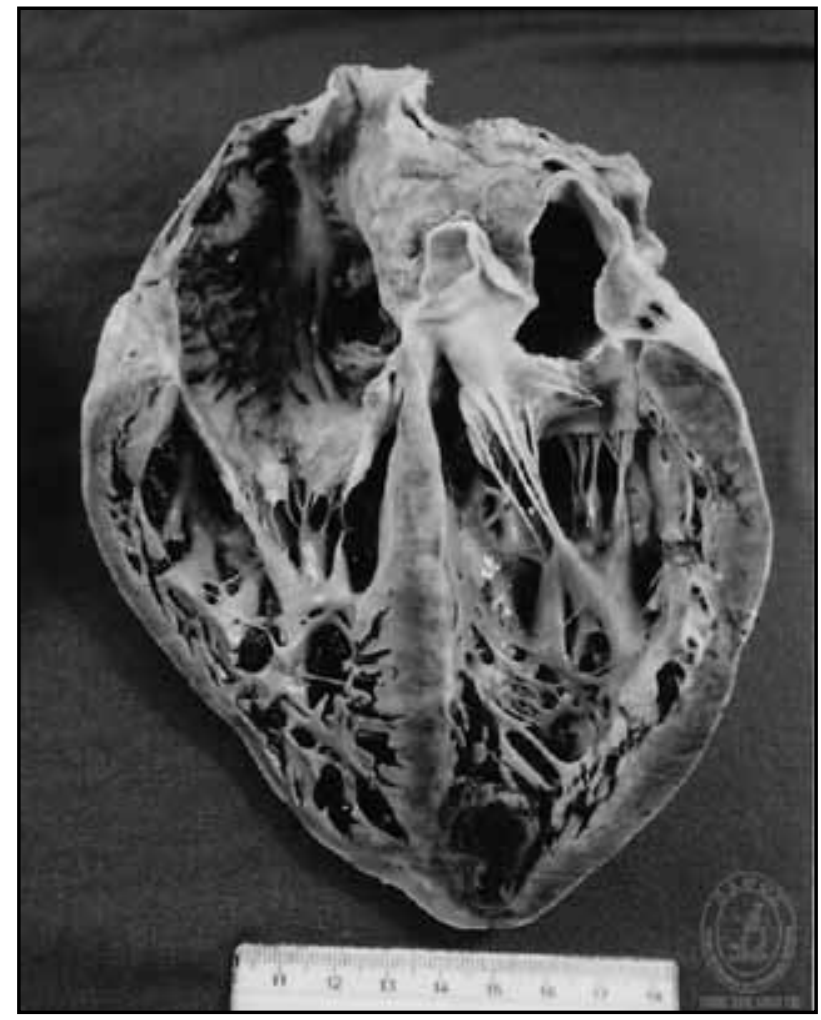

Figura 1 - Macroscopia

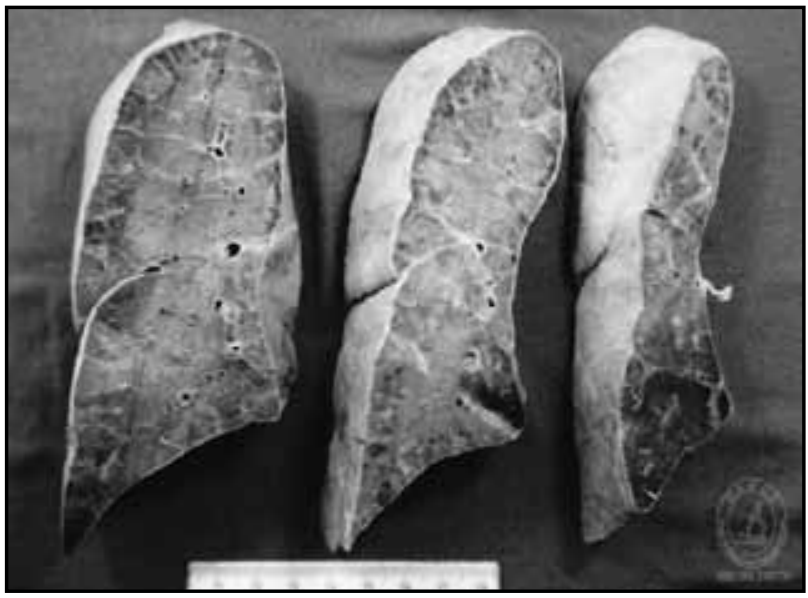

Figura 2 - Macroscopia 


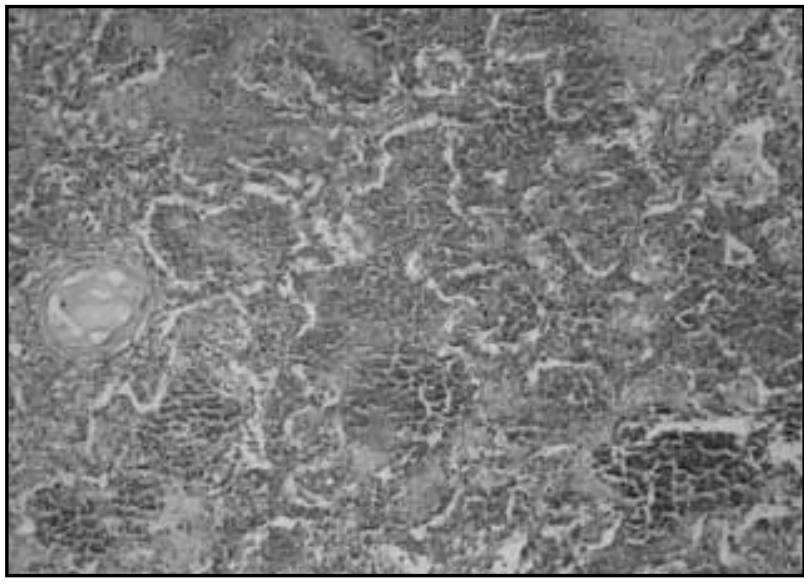

Figura 3 - Microscopia

e perivascular do miocárdio são evidentes, também mais proeminentes na zona subendocárdica. A análise histológica dos pulmões revelou extensa hemorragia alveolar.

Não obstante, o diagnóstico de infarto do miocárdio induzido pelo uso de cocaína é feito pela obtenção de história do quadro e exame físico do paciente, juntamente com testes laboratoriais apropriados, enzimas cardíacas e alterações eletrocardiográficas clássicas ${ }^{(2,5,7)}$. Todavia, nem sempre a apresentação clínica é clara, podendo haver sobreposição de quadros clínicos diferentes.

Nos pacientes que procuram o serviço de pronto atendimento médico com queixa de forte dor no peito de origem não traumática, a suspeita de uso de drogas ilícitas, especial- mente a cocaína, deve ser levantada. Em estudo de pacientes com queixa de dor no peito de origem não traumática, 17\% dos pacientes apresentaram cocaína ou seus metabólitos na urina, sendo que $77 \%$ destes encontravam-se na faixa etária entre 20 e 40 anos $^{(9)}$.

\section{Conclusões}

Embora a maneira com que alguns pacientes se apresentem permita diagnóstico claro, na grande maioria dos casos, será necessária investigação mais detalhada por meio da propedêutica complementar diagnóstica laboratorial. É extremamente importante identificar os pacientes com isquemia e infarto do miocárdio relacionados com o uso de cocaína, uma vez que as abordagens para o diagnóstico e tratamento são diferentes daqueles com doença cardíaca por aterosclerose.

Além disso, é essencial profunda compreensão do mecanismo fisiopatológico da doença para permitir o diagnóstico clínico de forma adequada e tratar essa potencialmente fatal e ainda comum condição.

Assim sendo, com o aumento da difusão da droga e a facilidade de acesso a ela, a cocaína deve ser adicionada à tradicional história epidemiológica, particularmente quando assistir a um jovem doente com queixa de dor torácica aguda, sabendo que esse é o sintoma mais comum em cocaína.

\section{Referências}

1. KLONER, R. A.; REZKALLA, S. H. Cocaine and the heart. N Engl J Med, v. 348, n. 6, p. 487-8, 2003

2. PITTS, W. R. et al. Cocaine-induced myocardial ischemia and infarction: pathophysiology, recognition and management. Prog Cardiovasc Dis, v. 40, n. 1, p. 65-76, 1997.

3. REZKALLA SH, KLONER RA. Cocaine-induced acute myocardial infarction. Clin Med Res, v. 5, n. 3, p. 172-6, 2007

4. COLEMAN, D. L.; ROSS, T. F.; NAUGHTON, J. L.Myocardial ischemia and infarction related to recreational cocaine use. West J Med, v. 136, n. 5, p. 444-6, 1982.

5. VONGPATANASIN, W.; LANGE, R. A.; HILLIS, L. D. Comparison of cocaine-induced vasoconstriction of left and right coronary arterial systems. Am J Cardiol, v. 79, n. 4, p. 492-3, 1997.

6. VILLOTA, J. N. et al. Cocaine-induced coronary thrombosis and acute myocardial infarction. Int J Cardiol, v. 96, n. 3, p. 481-2, 2004

7. BENZAQUEN, B. S.; COHEN, V.; EISENBERG, M. J. Effects of cocaine on the coronary arteries. Am Heart J, v. 142, n. 3, p. 402-10, 2001.

8. POZNER, C. N.; LEVINE, M.; ZANE, R. The cardiovascular effects of cocaine. J Emerg Med, v. 29, n. 2, p. 173-8, 2005.

9. HOLLANDER, J. E. et al.Chest pain associated with cocaine: an assessment of prevalence in suburban and urban emergency departments. Ann Emerg Med, v. 26, n. 6, p. 671-6, 1995. 